\title{
Effectiveness of Detection of Low Visibility Objects Via Multi-Sensor Intelligence Systems
}

\author{
Anton A. Koziratsky, \\ Ervand A. Mamajanyan and Andrei N. Shmarov* \\ Military Education and Research Centre of Military-Air Forces \\ «Military-Air Academy \\ Named After Professor N.E. Zhukovsky and Yu.A. Gagarin» \\ 54a Starykh Bolshevikov Str., Voronezh, 394064, Russia
}

Based on Dempster-Shafer evidence theory, a method was developed and analysis of effectiveness was implemented of low visibility objects detection with multi-sensor intelligence systems. Dependence of successful object detection probability from number of sensors in intelligence system was found. Their effectiveness and signal-to-noise ratio on output of optimal detector of each sensor.

Keywords: probability of successful object detection, multi-sensor intelligence systems, signal-tonoise ratio, Dempster-Shafer evidence theory.

Citation: Koziratsky A.A., Mamajanyan E.A., Shmarov A.N. Effectiveness of detection of low visibility objects via multisensor intelligence systems, J. Sib. Fed. Univ. Eng. technol., 2018, 11(1), 37-42. DOI: 10.17516/1999-494X-0005.

\section{Эффективность обнаружения малозаметных объектов мультисенсорными системами разведки}

А.А. Козирацкий, Е.А. Мамаджанян, А.Н. Шмаров Военныий учебно-научный цуентр Военно-воздушных сил «Военно-воздушная академия имени профессора Н.Е. Жуковского и Ю.А. Гагарина» Россия, 394064, Воронеж, ул. Старых Большевиков, 54а

На основе теории свидетельств Демпстера-Шефера разработана методика и выполнен анализ эффективности обнаружения объектов со сниженной заметностью мультисенсорными системами разведки. Установлень зависимости вероятности правильного обнаружения объектов от числа сенсоров в системе разведки, их

(c) Siberian Federal University. All rights reserved

* Corresponding author E-mail address: akoziratskiy@gmail.ru, Shmarov-an@mail.ru 
эффективности и отношения сигнал/шум на выходе оптимального обнаружителя каждого сенсора.

Ключевые слова: вероятность правильного обнаружения, мультисенсорная система разведки, отношение сигнал/шум, теория свидетельств Демпстера-Шефера.

Современные тенденции в развитии систем разведки состоят в комплексировании информации, получаемой разнородными средствами, работающими в различных спектральных диапазонах и на различных физических принципах [1]. Такие информационные системы называются мультисенсорными. Особое значение они приобретают в условиях информационного конфликта, когда системам разведки одной стороны противостоят системы разведки противоборствующей стороны и каждая из сторон имеет средства, препятствующие получению информации другой стороной. В этих условиях за счет комплексирования различных средств разведки сохраняется возможность получения необходимой информации в том случае, когда часть средств подавлена противоположной стороной, чем достигается выигрыш в конфликте [2]. В настоящее время в качестве одного из способов обеспечения выигрыша в конфликте рассматривается снижение заметности объектов [2], осуществляемое путем скрытия их демаскирующих признаков [3]. При создании малозаметных объектов, а также разработке систем их разведки целесообразно опираться на показатели, характеризующие эффективность разведки в зависимости от показателей эффективности входящих в них средств и параметров фоноцелевой обстановки.

Целью настоящей статьи является получение и анализ расчетных соотношений, устанавливающих связь показателей эффективности мультисенсорных систем разведки с показателями эффективности входящих в них сенсоров и параметрами фоноцелевой обстановки.

В современных системах разведки используются мультисенсорные системы с централизованной и децентрализованной архитектурой [4]. Оба типа систем состоят из нескольких каналов. Централизованная архитектура предполагает, что только единственный процессор обрабатывает все данные. В системе с децентрализованным построением каждый канал локально обрабатывает данные сенсора, проверяет их и проводит оценку состояния. Затем происходит обмен информацией между каналами (в виде оценок состояния или вероятностных характеристик) для ее уточнения на следующем этапе. В условиях жесткого дефицита времени, а также при неуверенном обнаружении объектов (обнаружении малозаметных объектов) предпочтение отдается системам разведки с централизованной архитектурой. Для обработки и принятия решения в них используются алгоритмы, основанные на теории свидетельств Демпстера-Шефера [5] (ТСДШ). По сравнению с методом Байеса [6], в котором рассматриваются априорные вероятности, в ТСДШ используется неклассическая идея «основной массы вероятности» как альтернатива традиционной вероятности $[4,5]$. Основная масса вероятности отражает начальное знание о системе, включая ее неопределенные состояния. Значение основной массы вероятности, обозначаемое $\mathrm{m}$, является основной мерой, характеризующей доверие гипотезы.

Пусть X - универсальное множество, рассматриваемых утверждений; $\mathrm{P}(\mathrm{X})$ - совокупность всех подмножеств множества $\mathrm{X}$, включая пустое множество. Масса $\mathrm{m}(\mathrm{x})$ элемента х множества $\mathrm{P}(\mathrm{X})$ выражает соотношение всех уместных и доступных свидетельств, которые поддержива-

$$
-38-
$$


ют утверждение, что определенный элемент х принадлежит $\mathrm{P}(\mathrm{X})$. Величина $\mathrm{m}(\mathrm{x})$ относится только к множеству $\mathrm{P}(\mathrm{X})$ и не создает никаких дополнительных утверждений о других подмножествах X, каждое из которых имеет свою массу.

$$
\begin{aligned}
& \mathrm{m}(\varnothing)=0 . \\
& \sum_{\substack{\mathrm{i}=1 \\
\mathrm{x}_{\mathrm{i}} \in \mathrm{P}(\mathrm{X})}}^{\mathrm{N}} \mathrm{m}\left(\mathrm{x}_{\mathrm{i}}\right)=1 .
\end{aligned}
$$

Здесь $\varnothing$ - пустое множество, $\mathrm{i}$ - номер элемента множества $\mathrm{P}(\mathrm{X}), \mathrm{i}=1 \ldots \mathrm{N}$.

Оцениваемыми состояниями для сенсоров многоканальной системы разведки являются: обнаружение объекта, обнаружение фона (т.е. отсутствие объекта) и неопределенное состояние, при котором информация, полученная сенсором, не позволяет отличить объект от фона. Меры доверия для каждого из оцениваемых состояний (с помощью М сенсоров различных спектральных диапазонов, входящих в состав многоканальной системы разведки) определяется в соответствии с правилом Демпстера $[4,5]$ :

$$
\mathrm{m}^{1,2 \ldots \mathrm{M}}(\mathrm{y})=\frac{\sum_{\mathrm{x}_{1} \cap \mathrm{x}_{2} \ldots \cap \mathrm{x}_{\mathrm{M}} \neq \varnothing} \prod_{1 \leq \mathrm{j} \leq \mathrm{N}} \mathrm{m}^{\mathrm{j}}\left(\mathrm{x}_{\mathrm{i}}\right)}{1-\mathrm{k}} ; \quad \mathrm{k}=\sum_{\mathrm{x}_{1} \cap \mathrm{x}_{2} \ldots \ldots \mathrm{x}_{\mathrm{M}}=\varnothing} \prod_{1 \leq \mathrm{j} \leq \mathrm{N}} \mathrm{m}^{\mathrm{j}}\left(\mathrm{x}_{\mathrm{i}}\right),
$$

где у - пересечение состояний $\mathrm{x}_{\mathrm{i}}$.

С помощью приведенного правила Демпстера (2) были исследованы характеристики обнаружения мультисенсорной системы разведки. В качестве оцениваемого показателя рассматривалась вероятность правильного обнаружения объекта в зависимости от количества сенсоров, входящих в систему разведки, и вероятностных характеристик обнаружения отдельного сенсора. Полученные результаты приведены на рис. 1 .

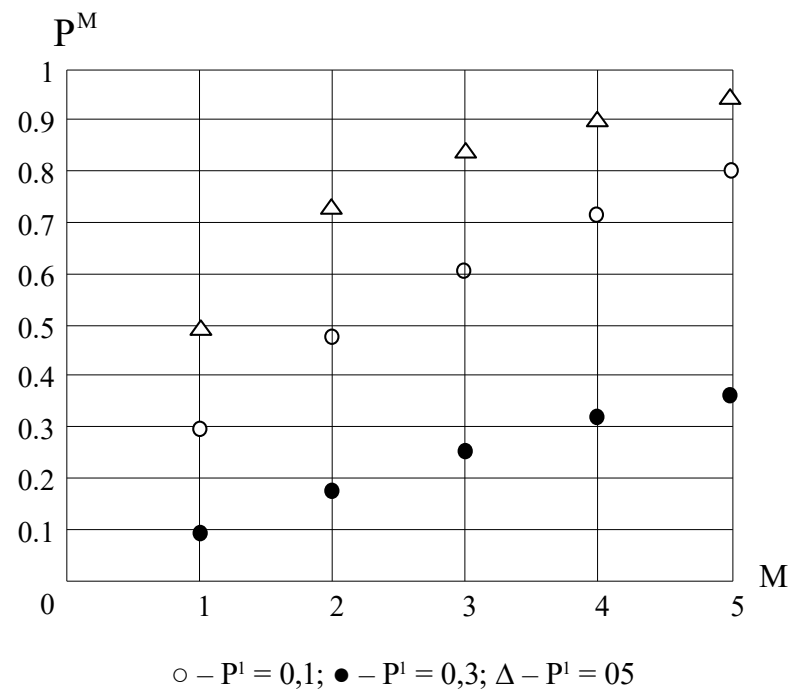

Рис. 1. Зависимость вероятности обнаружения объекта $\left(\mathrm{P}^{\mathrm{M}}\right)$ от количества сенсоров $(\mathrm{M})$ в системе разведки $1,2,3-\mathrm{P}^{\mathrm{l}}=0,1 ; 0,3 ; 05$

Fig. 1. Dependence of object detection probability $\left(\mathrm{P}^{\mathrm{M}}\right)$ on the number of sensors $(\mathrm{M})$ in the surveillance system $1,2,3-\mathrm{P}^{\mathrm{l}}=0,1 ; 0,3 ; 05$ 
Расчеты подтвердили очевидный результат, заключающийся в том, что для мультисенсорной системы вероятность правильного обнаружения объекта разведки растет с увеличением количества сенсоров, причем тем значительнее, чем выше вероятность обнаружения отдельного сенсора. Несмотря на очевидность данного вывода, полученные результаты позволяют определить требования к составу системы разведки и вероятностным характеристикам обнаружения входящих в нее средств. Для обеспечения вероятности правильного обнаружения объекта мультисенсорной системой разведки не менее 0,5 количество сенсоров должно быть не менее 3, а вероятность правильного обнаружения объекта отдельным сенсором должна быть не хуже 0,3 . Дальнейшее увеличение эффективности системы разведки возможно либо за счет увеличения числа сенсоров, либо за счет улучшения их характеристик обнаружения. Так, достижение вероятности правильного обнаружения объекта не менее 0,9 требует использования в системе разведки четырех сенсоров с вероятностью правильного обнаружения не менее 0,5 . При невозможности обеспечения вероятности обнаружения отдельного сенсора $\geq 0,5$ вероятность обнаружения объекта многоканальной системой разведки $\geq 0,9$ может быть обеспечена пятиканальной системой при условии вероятности обнаружения объекта отдельным сенсором не хуже чем 0,3 .

На рис. 2 представлены результаты исследований влияния параметров фоно-целевой обстановки (ФЦО) на характеристики обнаружения объекта мультисенсорной системой разведки. ФЦО в поле зрения отдельного сенсора задавалась с помощью параметра обнаружения q, равного отношению сигнал/шум по напряжению на выходе оптимального приемника локационного сигнала, имеющего случайные амплитуду и начальную фазу [7]. Исследования прово-

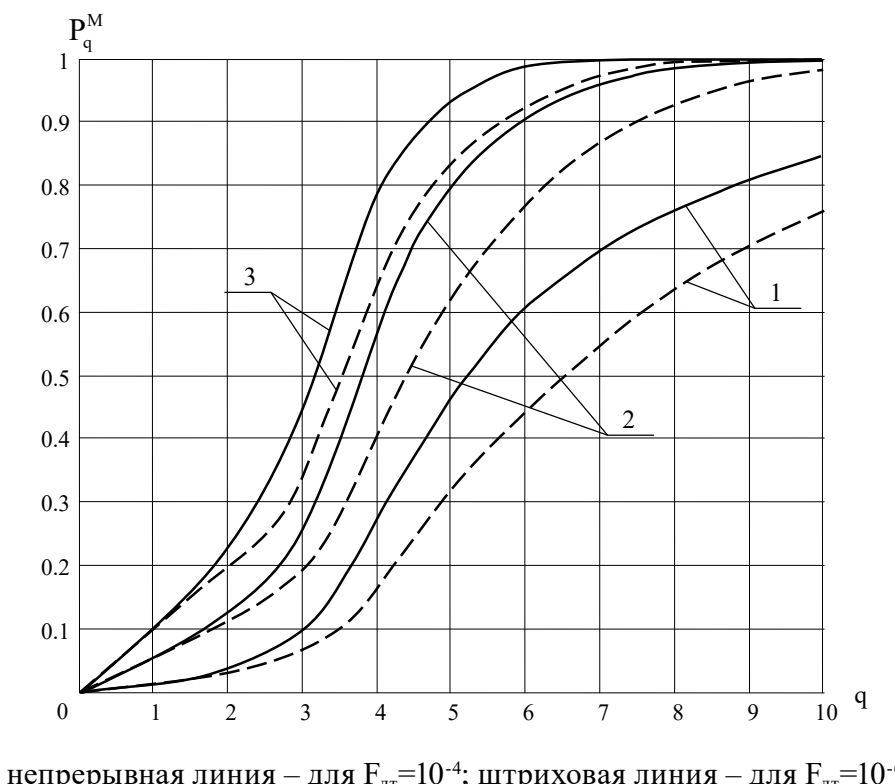

Рис. 2. Зависимость вероятности обнаружения объекта $\left(\mathrm{P}_{\mathrm{q}}\right)$ от величины параметра обнаружения (q) для различных значений вероятности ложной тревоги $\left(\mathrm{F}_{\text {лт }}\right) 1,2,3-\mathrm{M}=1,3,5$

Fig. 2. Dependence of object detection probability $\left(\mathrm{P}_{\mathrm{q}}\right)$ on the value of the detection parameter (q) for various values of false alarm probability $\left(\mathrm{F}_{\mathrm{fa}}\right) 1,2,3-\mathrm{M}=1,3,5$ 
дились по следующей методике. На выходе оптимального приемника в диапазоне $0 \div 10$ задавалась величина q, для которой с использованием [7] рассчитывалась вероятность правильного обнаружения сигнала отдельным сенсором $\mathrm{P}_{\mathrm{q}}^{\mathrm{M}=1}$ при вероятности ложной тревоги $\mathrm{F}_{\text {лт }}=10^{-4}$ и $\mathrm{F}_{\text {лт }}=10^{-6}$. Затем по значениям $\mathrm{P}_{\mathrm{q}}^{\mathrm{M}=1}$ с использованием правила Демпстера (2) рассчитывались значения вероятности обнаружения объекта мультисенсорной системой $\mathrm{P}_{\mathrm{q}}^{\mathrm{M}}=\mathrm{m}^{1,2, \ldots \mathrm{M}}(\mathrm{y})$. Полученные результаты приведены на рис. 2. Как и следовало ожидать, они повторяют результаты, отображенные на рис. 1. Вероятность $\mathrm{P}_{\mathrm{q}}^{\mathrm{M}}$ тем больше, чем больше значения q и М. Вместе с тем, они позволяют определить требования к защищенности объекта от обнаружения мультисенсорной системой разведки. Для обеспечения вероятности правильного обнаружения объекта системой разведки, включающей 1,3 и 5 сенсоров, не более 0,2 отношение сигнал/шум на выходе оптимального приемника в каждом из каналов не должно превышать значений 3,$6 ; 2,7$ и 1,8 для $\mathrm{F}_{\text {лт }}=10^{-4}$ и 4,$2 ; 3,1$ и 2 для $\mathrm{F}_{\text {лт }}=10^{-6}$.

\section{Заключение}

Таким образом, в настоящей работе разработан научно-методический аппарат, устанавливающий связь показателей эффективности мультисенсорных систем разведки с показателями эффективности входящих в них сенсоров и параметрами фоноцелевой обстановки. Он позволяет определять требования к составу систем разведки, вероятностным характеристикам обнаружения входящих в них средств, а также требования к защищенности объектов от обнаружения мультисенсорными системами разведки.

\section{Список литературы}

[1] Меньшаков Ю.К. Теоретические основы технических разведок: учеб. пособие. Под ред. Ю.Н. Лаврухина. М.: Изд-во МГТУ им. Н.Э. Баумана, 2008. 536 с: ил. [Menshakov Yu.К. Theoretical Fundamentals of Technical Surveillance, training manual, under the editorial supervision of Lavrukhin Yu.N., BMSTU Publishing House, 2008, 536 p. (in Russian)]

[2] Модели информационного конфликта средств поиска и обнаружения. Под ред. Ю.Л. Козирацкого. М.: Радиотехника. 2013. 232 с. [Models of Information Mismatch of Means of Search and Detection/ under the editorial supervision of Koziratski Yu.L., Radiotekhnika, 2013, 232 p. (in Russian)]

[3] Понькин В.А. Оптическая заметность летательных аппаратов. Под ред. В.А. Понькина и Э.В. Петещенкова. Воронеж. «Научная книга». 2015. 553 с. [Ponkin V.A. Optical Visibility of Aircraft, under the editorial supervision of Ponkin V.A. and Petaschenkov E.V., Voronezh, "Nauchnaya Kniga", 2015, 553 p. (in Russian)]

[4] Андрющенко М.С., Степанов В.В. Обработка информации в мультисенсорных системах. Вооружение и экономика. 2015, 4, 39-45. [Andryuschenko M.S., Stepanov V.V., Information Processing in Multisensor Systems. Weapons and Economics, 2015, 4, 39-45 (in Russian)]

[5] Ганцева Е.А., Каладзе В.А. Статистический подход разрешения неопределенности экспертных суждений на основе теории случайных множеств. Вестник ВГУ. Серия: системный анализ и информационные технологии. 2015, 2, 83-88. [Gantseva E.A., Kaladze V.A., Statistical Approach to Resolution of Ambiguity of Expert Judgements Based on the Theory of Random Sets, Vestnik VGU, series: Systemic Analysis and Information Technologies, 2015, 2, 83-88 (in Russian)] 
[6] Теория обнаружения сигналов. Под. ред. П.А. Бакута. М., Сов. радио, 1984 [Theory of Signal Detection, under the editorial supervision of P.A. Bakut M., Sov. Radio, 1984 (in Russian)]

[7] Ширман Я.Д., Голиков В.И. Основы теории обнаружения радиолокаиионных сигналов и измерения их параметров. М.: Сов. радио. 1963. 278 с. [Shirman Ya.D., Golikov V.I. Bases of Theory of radar Signal Detection and Measurement of their Parameters, So. Radio, 1963, 278 p. (in Russian)] 